\title{
Fabrication of multi-scale periodic surface structures on Ti-6Al-4V by direct laser writing and direct laser interference patterning for modified wettability applications
}

\author{
D. Huerta-Murillo ${ }^{\text {a,* }}$, A.I. Aguilar-Morales ${ }^{\text {b }}$, S. Alamri ${ }^{\text {b }}$, J.T. Cardoso ${ }^{\text {a }}$, R. Jagdheesh ${ }^{\text {, }}$, \\ A.F. Lasagni ${ }^{b, c}$, J.L. Ocaña ${ }^{a}$ \\ ${ }^{a}$ UPM Laser Centre, Polytechnical University of Madrid, Ctra. Valencia km 7.3, 28031, Madrid, Spain \\ ${ }^{\mathrm{b}}$ Fraunhofer-Institut für Werkstoff- und Strahltechnik IWS, Winterbergstr. 28, 01277 Dresden, Germany \\ ${ }^{\mathrm{c}}$ Institut für Fertigungstechnik, Technische Universität Dresden, George-Bähr-Str. 3c, 01062 Dresden, Germany
}

\section{A R T I C L E I N F O}

\section{Keywords:}

Laser material processing

Ti-6Al-4V

Direct laser writing

Direct laser interference patterning

Laser-induced periodic surface structures

\begin{abstract}
A B S T R A C T
In this work, hierarchical surface patterns fabricated on Ti-6Al-4V alloy combining two laser micro-machining techniques are presented. The used technologies are based on nanosecond Direct Laser Writing and picosecond Direct Laser Interference Patterning. Squared shape micro-cells with different hatch distances were produced by Direct Laser Writing with depths values in the micro-scale, forming a well-defined closed packet. Subsequently, cross-like periodic patterns were fabricated by means of Direct Laser Interference Patterning using a two-beam configuration, generating a dual-scale periodic surface structure in both micro- and nano-scale due to the formation of Laser-Induced Periodic Surface Structure after the picosecond process. As a result a triple hierarchical periodic surface structure was generated. The surface morphology of the irradiated area was characterized with scanning electron microscopy and confocal microscopy. Additionally, static contact angle measurements were made to analyze the wettability behavior of the structures, showing a hydrophobic behavior for the hierarchical structures.
\end{abstract}

(C) 2017 Elsevier Ltd. All rights reserved.

\section{Introduction}

Lasers have been widely used on several technological fields over the last decades, including medical and industrial applications. In the case of the engineering area, a great variety of laser-based devices with specific tasks have been created. Particularly, laser micromachining systems for fast and precise material processing have been developed. The laser micro-machining process can be done by means of different techniques, which differ from each other in the laser source parameters or the optical path and components for the laser beam delivery system. It has been shown that different materials can be patterned in the nano- and micro-scale using pulsed laser irradiation, creating diverse geometrical shapes and enhancing the properties of the material such as increased optical absorptance on micro-structured Si [1], improved photoelectron emission current from nanostructured platinum surfaces [2] or super-hydrophobic surfaces on micro-structured aluminum sheets [3], to mention a few. The physical properties of these micro-structures strongly depend on the parameters used during the laser material processing as well as the intrinsic properties of the laser-matter interaction and the treatment atmosphere. Therefore, the feasibility in processing a material translates into the possibility to create complex structures and, in some cases, to reproduce topographies present in plants like the lotus leaves or rose petals [4-6] or on insect skin [7-10], that exhibit high hydrophobic behavior. In this context, bio-inspired hierarchical surface structured materials created with several techniques have shown several improvements with respect to wettability [11-13] or better resistance to corrosion [14-16]. For this reason, hierarchical periodic structures fabricated by laser based technologies have been studied for enhanced functionalization on metallic surfaces [17-19].Titanium and its alloys, which it is one of the most suitable metallic material for biomedical applications $[20,21]$, have demonstrated the possibility of creating surfaces with like self-cleaning effects [22], improved biocompatibility [23] or enhanced cell attachment [24,25].

In this work, an experimental study of the fabrication of hierarchical structures on Ti-6Al-4V substrates, combining two laser micromachining techniques with two different pulse durations: nanosecond Direct Laser Writing and picosecond Direct Laser Interference Patterning is presented. Furthermore, Laser-Induced Periodic Surface Structure

\footnotetext{
* Corresponding author.

E-mail address: d.huerta@upm.es (D. Huerta-Murillo).
} 


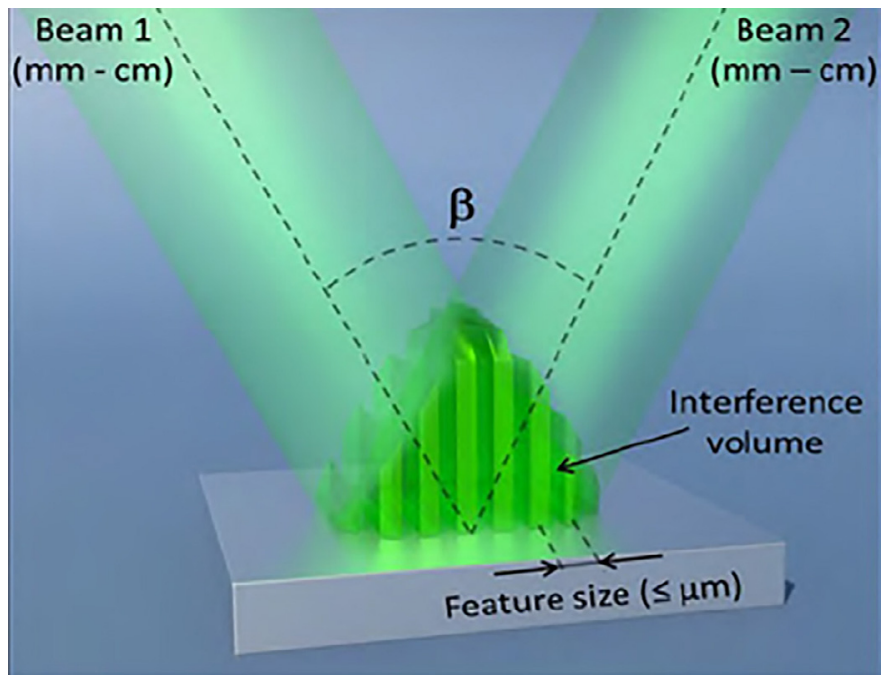

Fig. 1. Schematic for the two-beams Direct Laser Interference Patterning ( $\beta$ corresponds to the angle between the laser beams and controls together with the laser wavelength the resulting spatial period).

(LIPSS) were also produced during the picosecond micro-machining process resulting in a triple hierarchical periodic surface structure (microand nano-scale features). The structures were analyzed by means of Scanning Electronic Microscopy (SEM) and Confocal Microscopy (CM). Also, static contact angle measurements were carried out to analyze the wettability behavior of the structures.

In the laser micro-machining techniques, a controlled amount of material is removed from the bulk sample via laser ablation. The ablation, which occurs when the laser fluence is sufficient to break the materials bonds, can be mainly photo-thermal or photo-chemical depending on the laser pulse duration, wavelength and on the material properties (absorption coefficient, electronic structure). For the case of laser-structuring on metals employing nanosecond pulses, the ablation is mainly thermal and the structuring is controlled by the Marangoni convection, which describes how the melted material flow behaves. In the Direct Laser Writing (DLW) technique, the laser beam is focused directly on the sample surface and the relative motion of the beam with respect to the workpiece allows creating the desired surface structure by means of controlled local ablation. DLW has demonstrated a wide range of applications like 2D direct laser engraving [26], machining micro patterns on the surface of a photopolymers for 3D micro-structuring [27], micromachining of graphene oxide that can be reduce to micro-patterned graphene nano-sheets [28], or micro-drilling of aluminum sheets to fabricate super-hydrophobic surfaces [29]. Recently, a efficacious microstructures known as $\mu$-cell have been proved to enhance the wettability behavior of aluminum sheets surfaces, making possible the fabrication of super and near-ultra hydrophobic surfaces with self-cleaning properties and highly bouncing effect by means of direct laser writing [30]. Nevertheless, hard limitations are encountered for this laser micro-machining technique when the fabrication of features smaller that the beam size are required, since the resolution of the technique is typically limited to the dimensions of the focused beam, making necessary the use of other techniques to create structures with smaller resolution.

Direct Laser Interference Patterning (DLIP) is nowadays one of the most effective techniques for texturing materials up to the submicrometer level, with high quality and precision [31-33]. The DLIP process employs the interference generated by coherently overlapping two or more laser beams and thereby, producing a periodic modulation of the laser intensity. The shape of the interference pattern is directly transferred to the material surfaces and depending on the number of beams used different geometries are possible. In the case of two beams (Fig. 1), the interference pattern consist on a line-like geometry, with a characteristic spatial period $\Lambda$, which can be calculated using Eq. (1):

$\Lambda=\lambda / 2 \sin (\beta)$

where $\beta$ is the angle between the laser beams and $\lambda$ is the laser wavelength. Thus, the materials can be locally ablated at the interference maxima positions, depending on the laser-matter interaction conditions. Periodic surface patterns can be produced in a one-step process with controllable pitch and geometry, allowing the creations of lines, pillars, holes and other geometries [34-38], both in ablation and swelling regimes [39]. This characteristic has allowed DLIP to directly process different surfaces for a wide number of application fields such as increasing the optical transmittance of Indium thin oxides [40], enhance tribological performances of ta-C thin films [41], fabricating micro-lenses [42], patterning of graphene oxide for humidity sensing [43], scaffold fabrication for tissue engineering [44] or surface structuring of biocompatible diamond-like carbon layers for controlled cell growth $[45,46]$. Moreover high fabrication speeds have been recently reported both for polymers [47] and metals [48].

Among the different types of surface structures that have been reported after pulsed laser irradiation of several materials, Laser-Induced Periodic Surface Structures (LIPSS) have attracted high attention in the field of surface functionalization [49]. These structures consist of elongated ripples or grooves with a typical height of several nanometers and a characteristic periodicity near (Low Spatial Frequency LIPSS, LSFL) or much lower (High Spatial Frequency LIPSS, HSFL) than the laser wavelength used for the laser processing [50]. The formation of LIPSS has been observed under different irradiation conditions on different materials (semiconductors, dielectrics and metals) with a direction of orientation either parallel (HSFL) [51] or perpendicular to the laser polarization direction (LSFL) [52]. Although several theories has been proposed trying to explain the formation process of LIPSS [53-57], the physical mechanism of LIPSS formation is not completely experimentally clear.

\section{Experimental}

\subsection{Material}

In this study, $2 \mathrm{~mm}$ thick samples of Ti-6Al-4V have been used for laser surface micro-structuring by means of DLW and DLIP. The material has been processed without any prior process, with the exception of a cleaning with ethanol preceding the laser processing. The samples were kept on air atmosphere and no further treatment was made after the laser processing. The surface morphology of the sample was examined by scanning electron microscopy (SEM HITACHI S-3000N) and confocal laser scanning microscopy (Leica DCM 3D). Moreover, static contact angle measurements were carried out to analyze the wettability behavior of the structures.

\subsection{Direct laser writing}

The experiments were carried out in ambient environment using a Qswitched pulsed Spectra-Physics nanosecond solid-state laser source that generates pulses with a fixed duration of 15 nanoseconds, at a repetition rate of $20 \mathrm{kHz}$, with a central wavelength of $1064 \mathrm{~nm}$. The fundamental wavelength is frequency-tripled by a nonlinear optical conversion to obtain pulses with a wavelength of $355 \mathrm{~nm}$. The laser beam was focused onto the sample at normal incidence with a $250-\mathrm{mm}$ focal lens to generate a spot size of $15 \mu \mathrm{m}$. The position of the sample was controlled using a translational stage with a resolution of $0.1 \mu \mathrm{m}$ to create squared shaped micro-cells, by using a fixed scan speed of $10 \mathrm{~mm} / \mathrm{s}$. The distance between each micro-cell is labeled as Hatch Distance (H.D). The process parameters used for the fabrication of the micro-cells are shown in Table 1. 
Table 1

Laser processing parameters for the fabrication of micro-cells on Ti-6Al-4V via DLW technique.

\begin{tabular}{ll}
\hline Avg. laser power $(\mathrm{mW})$ & $100-280$ \\
Repetition rate $(\mathrm{kHz})$ & 20 \\
Scan speed $(\mathrm{mm} / \mathrm{s})$ & 10 \\
Hatch distance (H.D.) $(\mu \mathrm{m})$ & $30-50$ \\
Beam spot size $(\mu \mathrm{m})$ & 15 \\
\hline
\end{tabular}

Table 2

Laser processing parameters for the DLIP technique.

\begin{tabular}{ll}
\hline Avg. laser power $(\mathrm{mW})$ & 162 \\
Fluence $\left(\mathrm{J} / \mathrm{cm}^{2}\right)$ & 0.8 \\
Repetition rate $(\mathrm{kHz})$ & 1 \\
Interference spatial period $(\mu \mathrm{m})$ & $1.1,2.6$ and 5.4 \\
Interference area diameter $(\mu \mathrm{m})$ & 160 \\
\hline
\end{tabular}

\subsection{Direct laser interference patterning}

The second laser micro structuring process was performed using Direct Laser Interference Patterning with a Q-switched picosecond laser (EDGEWAVE solid-state Innoslab Nd: $\mathrm{YVO}_{4}$, pulse duration: $10 \mathrm{ps}$ ) at the fundamental wavelength of $1064 \mathrm{~nm}$. The repetition rate was fixed at $1 \mathrm{kHz}$. For the DLIP set-up, the laser beam is split in two sub-beams and then overlapped onto the substrate (Fig. 1). The patterned area corresponds to the superposition zone of the beams which was in our case approximately $160 \mu \mathrm{m}$. For a two beam DLIP system, the interference patter has a line-like intensity distribution. A second irradiation perpendicularly to the first pattering with an angle of $90^{\circ}$ allowed generating a periodic pillar-like pattern on top of the prior structures created via DLW and thus creating a dual-scale periodic surface structures. The process parameters used are shown in the Table 2.

\section{Results and discussion}

The micro-fabrication of hierarchical periodic surface structures on Ti6Al4V substrate has been carried out by means of a two-technique laser surface structuring process, using nano- and picosecond laser sources. The DLW technique has been used to produce structures in the micro-range (due to the beam-size dimensions). Micro- and nanostructures produced by DLIP were fabricated over the prior produced DLW structures. The final pattern is a hierarchical periodic surface structure.

\subsection{Direct laser writing (DLW) structures}

In the first micro-structuring process, square-shape micro-structures with five different hatch distance have been produced with the process parameters shown in Table 1. For all used laser powers, the DLW process allowed to create well-defined periodic surface structures. Fig. 2a shows a Scanning Electron Microscope (SEM) image of one the processed surface areas (with wavelength: $355 \mathrm{~nm}$, pulse duration: $15 \mathrm{~ns}$, repetition rate: $20 \mathrm{kHz}$, laser fluence: $5.65 \mathrm{~J} / \mathrm{cm}^{2}$, scan speed: $80 \mathrm{~mm} / \mathrm{s}$ ). Typical thermal effects that occur during a nanosecond laser process are appreciated, where recast formation of the molten material has taken place. During the laser ablation, the molten material recast as the laser scan the sample, inducing the formation of micro-walls around the original surface forming a closed packet or $\mu$-cell. In addition, also some residual granular particles are produced due to the solidification of the molten material. These DLW structures generate interest due to the potential application on enhancing the wettability behavior of the titanium alloy by means of a micro-structured surface as it has been showed that a $\mu$ cell type structure can be used to create a super-hydrophobic surface on Aluminum alloys [3]. In addition to the characterization by SEM, the laser irradiated areas were also analyzed by confocal microscopy. Fig.
$2 \mathrm{~b}$ shows an example of a cross-sectional profile of the fabricated $\mu$-cell from Fig. 2a.

The dimensions of these micro-structures depend strongly on the power used for the ablation of the micro-channels. Fig. 3 shows the relation between the hatch distances and the height of the walls of the $\mu$-cell for four different avg. pulse power. As the power increases, the fluence over the surface area increase (due to the spot size remaining constant). Thus, the material is removed in a higher extent inducing the recast on the top while also re-filling the patterned micro-channel with the molten material as the laser scan the material during the laser processing. This can be observed by noticing that the horizontal and vertical micro-channels are not created in a symmetric way, as one of them is open in the direction of the laser writing while the other (the first one made) is blocked due to the recast of material as the perpendicular channels are made. This effect also affects the volume (width and height) of the recast layer of molten material, i.e., the $\mu$-cell. For low avg. power values, the amount of ablated material is lower and well-defined structures are produced, therefore, the recast of the molten material is smaller, thus reflexing in lower altitude of the micro-walls. As the avg. laser power increases, more recast material starts to accumulate, giving place to higher walls, but less defined structures, due to the amount of uncontrolled recast. As the power increases, the amount of molten material is higher, thus growing the volume of recast material and the height of the micro-wall, reaching a maximum value of more than $5 \mu \mathrm{m}$ for a Hatch Distance of $40 \mu \mathrm{m}$. Additionally, it can be observed that microwalls' height is influenced by the periodic distance between channels. When the distance is lower, there is an accumulation of recast material on adjacent walls, thus increasing height values. However, during the scanning process, perpendicular ablation to the direction of the previously formed walls decreases their height as the material is melted again. For higher hatch distances, there is less accumulation of recast material on adjacent walls, due to the large gap between the structures. The highest wall height values where measured for $40 \mu \mathrm{m}$ of Hatch Distance, where the periodic gap promotes both accumulation of recast and fewer ablation of previously formed micro-walls.

\subsection{Direct laser interference patterning (DLIP) structures}

To study the fabrication of DLIP micro-structures on Ti-6Al-4V, irradiations with a picosecond laser were performed using the parameters shown in Table 2 . Once the laser parameters are fixed, line-like patterns with three different spatial periods $(1.1 \mu \mathrm{m}, 2.6 \mu \mathrm{m}$ and $5.4 \mu \mathrm{m})$ have been produced using the two-beam configuration. The structuring has been performed employing high pulse-to-pulse overlap in the direction of the interference lines. A detailed description of the process has been reported elsewhere [58]. Sequential experiments have been carried out considering an interference area of $160 \mu \mathrm{m}$ while decreasing the pulse-to-pulse distance from $144 \mu \mathrm{m}$ to $4.8 \mu \mathrm{m}$, in the direction of the interference lines, while the laser fluence was kept constant with a value of $0.8 \mathrm{~J} / \mathrm{cm}^{2}$. Fig. 4 depicts the relation between overlap used and the depth produced on the surface by DLIP for a line-like pattern and it can be appreciated the rapidly increase in depth for the spatial periods of $5.4 \mu \mathrm{m}$ and $2.6 \mu \mathrm{m}$ in the range of $80-97 \%$. It can be seen that for the largest used spatial period the achieved depth was $2.5 \mu \mathrm{m}$, while for the spatial period of $1.1 \mu \mathrm{m}$ the increase is barely noticed in comparison with the other two periods. The difference in depth may be related to the accumulation of heat for a large number of pulses. This effect is stronger for smaller periods due to the reduced distance between each interference maxima, leading to a pattern partially destroyed.

In a subsequent process in order to create the cross-like structure, the sample has been rotated $90^{\circ}$ and irradiated again with the same value of overlap but with a change in the fluence. In this set of experiments, it was determined to reduce the fluence to $0.55 \mathrm{~J} / \mathrm{cm}^{2}$ in the second irradiation, because homogenous structures have been measured according with the process of high overlap followed [58]. In Fig. 5 are shown the measurements obtained using confocal microscopy of the cross-like 

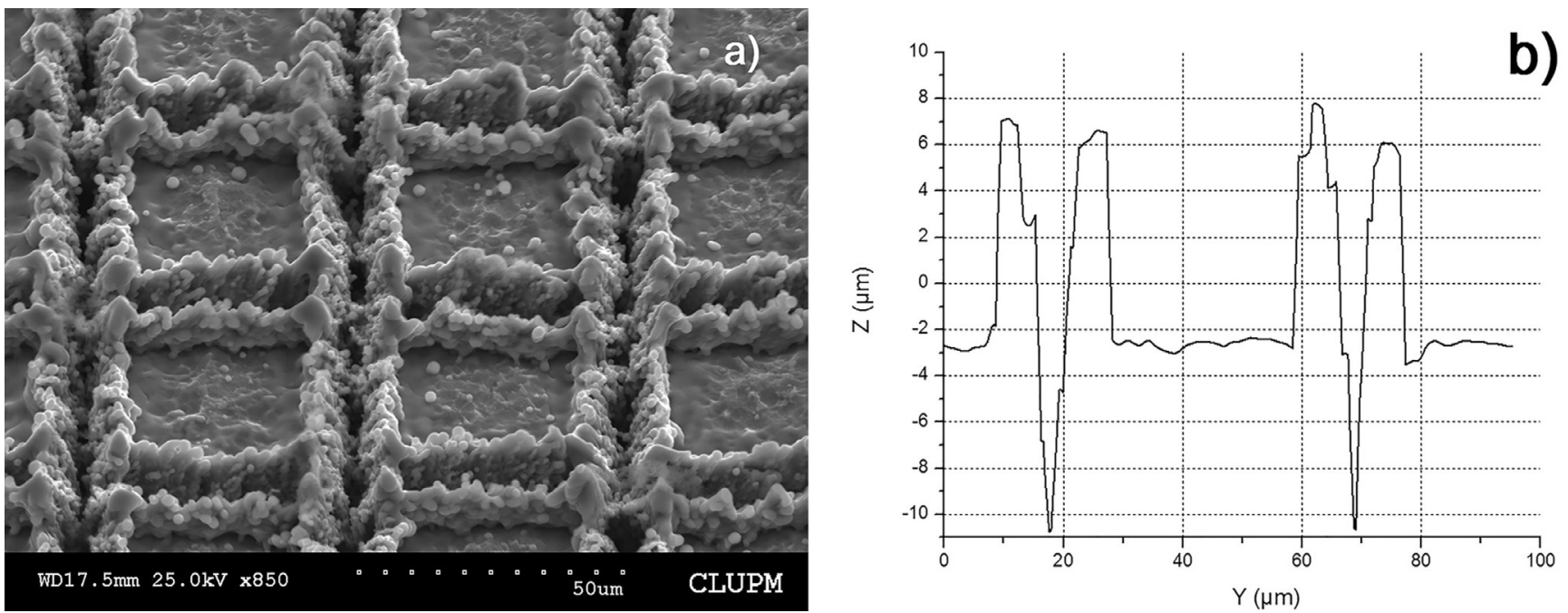

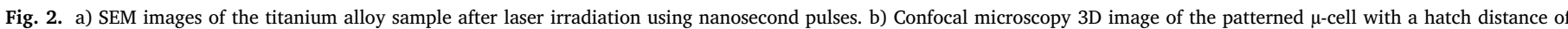
$50 \mu \mathrm{m}$ (wavelength: $355 \mathrm{~nm}$, pulse duration: $15 \mathrm{~ns}$, repetition rate: $20 \mathrm{kHz}$, laser fluence: $5.65 \mathrm{~J} / \mathrm{cm}^{2}$, scan speed: $80 \mathrm{~mm} / \mathrm{s}$ )

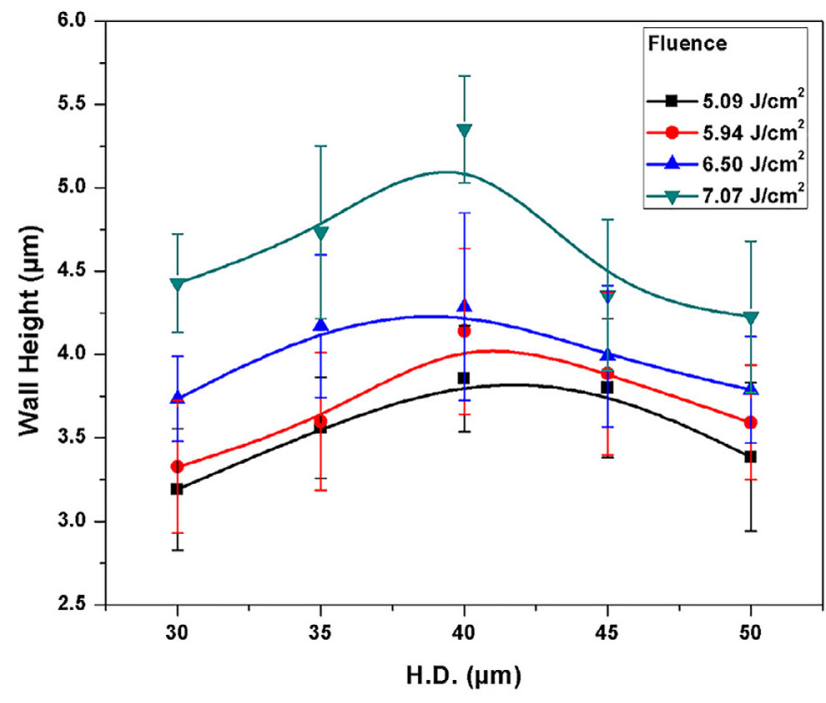

Fig. 3. Wall height values for four different avg. pulse powers $(180-250 \mathrm{~mW})$ as function of the hatch distances $(30-50 \mu \mathrm{m})$.

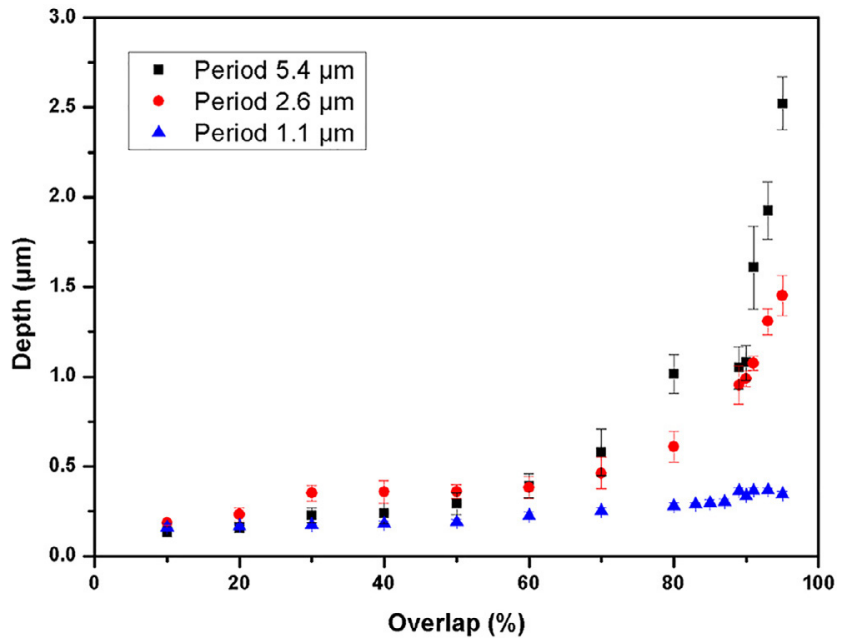

Fig. 4. Relation between overlap used during the DLIP in the two-beam configuration and the average depth produced (wavelength: $1064 \mathrm{~nm}$, pulse duration: $10 \mathrm{ps}$, repetition rate: $1 \mathrm{kHz}$, Fluence: $0.8 \mathrm{~J} / \mathrm{cm}^{2}$ ).

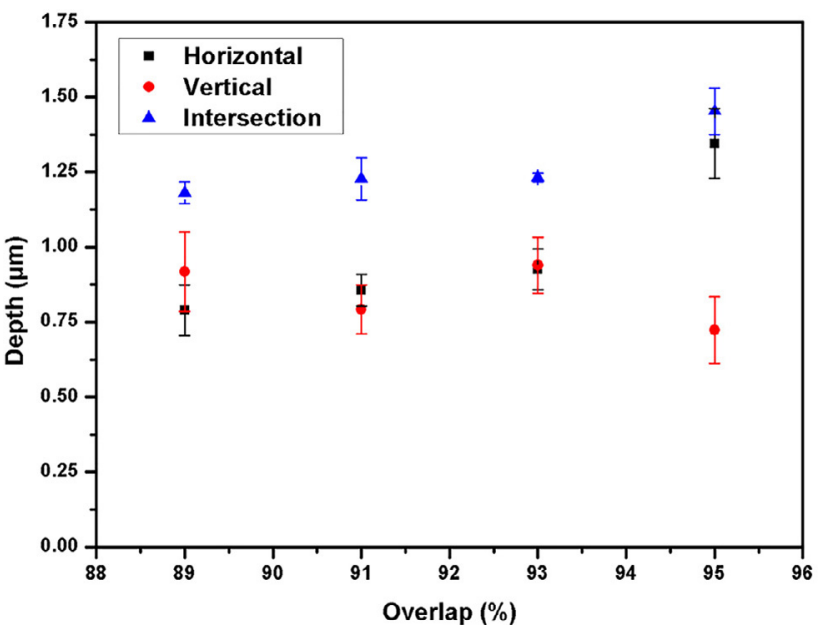

Fig. 5. Depths measured in the cross-like structures on Ti-6Al-4V using different high values of overlap in the spatial period of $2.6 \mu \mathrm{m}$ (wavelength: $1064 \mathrm{~nm}$, pulse duration: $10 \mathrm{ps}$, repetition rate: $1 \mathrm{kHz}$, fluence for the second irradiation: $0.55 \mathrm{~J} / \mathrm{cm}^{2}$ ).

structures produced with a spatial period of $2.6 \mu \mathrm{m}$. Both the structure depths in the horizontal and vertical directions as well as the intersection points are depicted. For all overlap values the depth of the cross-like was less than $1 \mu \mathrm{m}$ with very close values for the horizontal and the vertical directions (superposition of the interference minima and maxima) except for the case of $95 \%$ pulse overlap. Also a trend can be noticed related with the increment of the depth in the intersections (superposition of interference maxima) in relation with the overlap used.

An example of a treated surface with micro-pillars with a spatial period of $2.6 \mu \mathrm{m}$ can be observed in Fig. 6a. This structure was fabricated using an overlap of $93 \%$. The surface structure generated by the DLIP technique is uniform all over the processed area, showing a periodic set of micro-pillars with an average depth of $0.9 \mu \mathrm{m}$ at both the vertical and horizontal direction and an average depth of $1.5 \mu \mathrm{m}$ at the interference maxima-maxima intersection.

Moreover, during the processing of Ti-6Al-4V with the DLIP technique, the formation of LIPSS could be also observed at the interference maxima positions, giving place to micro-pillars with a rough top surface and thus producing in a one step process a dual-scale or hierarchical structures (nano and micro features) as shown in Fig. 6a. The micro-pillars exhibit a hierarchical structure that may be suitable for 

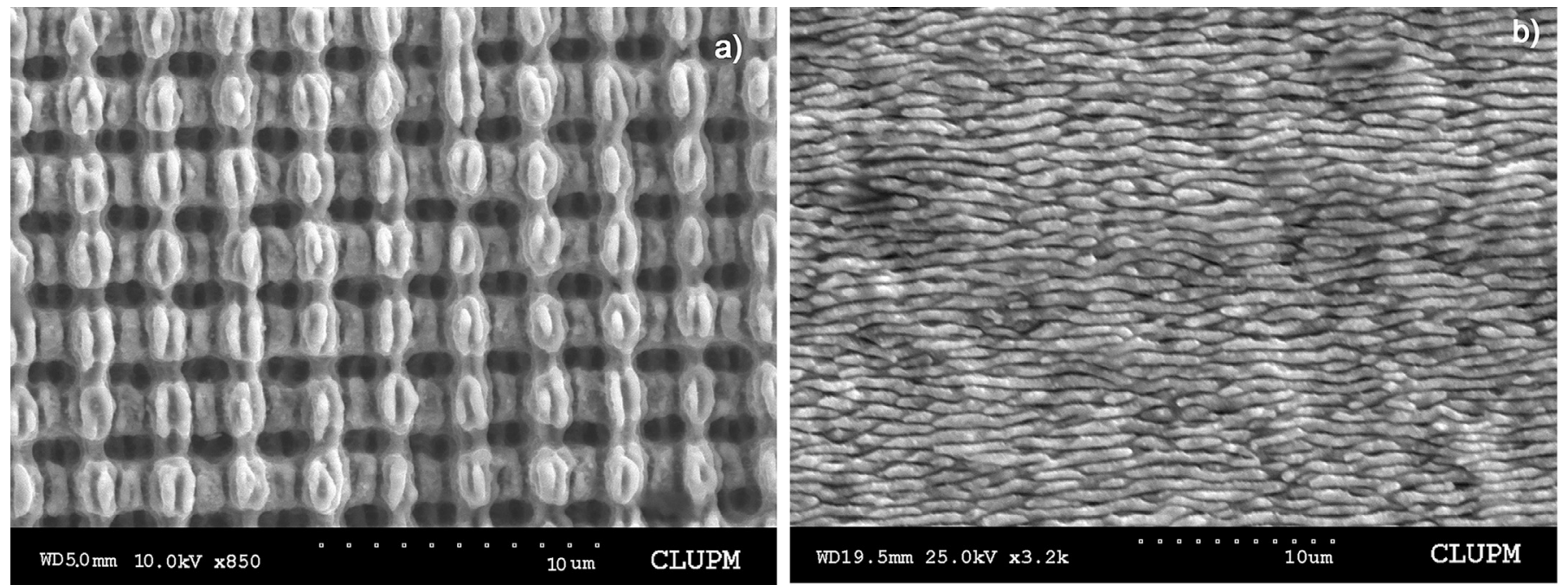

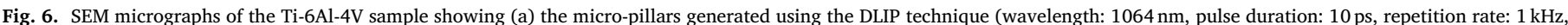
fluence for the second irradiation: $0.55 \mathrm{~J} / \mathrm{cm}^{2}$, spatial period: $2.6 \mu \mathrm{m}$.) and (b) laser induced periodic surface structures (LIPSS) produced over the DLIP pattern.

wettability applications. According to Skolski et.al [49], the produced LIPSS (Fig. 6b) can be classified as Low Spatial Frequency LIPSS (LSFLs or type-s). Thus, the direction of the LSFLs is perpendicular to the direction of the laser polarization. The observed spatial period has a value of $790 \mathrm{~nm} \pm 70 \mathrm{~nm}$, which is slightly smaller than the used laser wavelength $(1060 \mathrm{~nm})$

\subsection{Combined-process structures}

Because of this difference of spatial resolution between the two previous techniques, the combination of both techniques has been investigated. For this purpose, firstly squared shaped micro-cells were generated by means of DLW with nanosecond pulses using the parameters reported before for a hatch distance of $50 \mu \mathrm{m}$. After that, a pillar-like pattern was produced using DLIP with a spatial period of $2.6 \mu \mathrm{m}$ sing the process parameters from the previous section. The result can be observed in Fig. 7a and b where the triple hierarchical structures (nanoand micro-features) generated by the combination of both techniques are visible. A cross-section profile of the obtained surface pattern is shown in Fig. 7c.

The micrograph shows clearly that the second process with DLIP does not damage the previously fabricated $\mu$-cell using DLW. In the center of the $\mu$-cell, where the original topography of the sample along with some recast particles was observed before, a well-defined periodic structure has been fabricated. The results obtained for the fabrication of two different types of micro-structures generated with the two micro-machining techniques shows that, the DLIP technique can reach a smaller spatial resolution between adjacent micro-structures in comparison with the resolution of the DLW technique which is limited by the spot size of the laser beam, thus giving place to a dual scale roughness surface. Additionally, the formation of LIPSS during the DLIP process gives the tools necessary for creating a triple scale roughness surfaces.

\subsection{Wettability analysis}

A preliminary analysis have been made to see the effect of the $\mu$-cell on the wetting properties of the Ti-6Al-4V by static contact angle (SCA) measurements, with a droplet size of $3 \mu \mathrm{l}$. The non-patterned sample was measured as a reference and recorded a SCA value of $67 \pm 2^{\circ}$ (Fig. 8 a). The DLW patterned samples were hydrophilic after the laser processing, reaching a SCA value of $91.3^{\circ}$, slightly improving the wetting properties of the material, as seen on Fig. $8 \mathrm{~b}$, therefore the $\mu$-cell structures by themselves did not increase in a well manner the wettability behavior of the Ti alloy samples. The lightly increase in the contact angle may be prompted due to the $\mu$-cells patterned, as it has been probed on aluminum alloy samples [3] where it has been demonstrated that the C.A. depends on the hatch distance, showing a decrease in the C.A as the separation increases. The $\mu$-cell act as air traps that prevent the surface from wetting reducing the area of liquid-solid interface, thus a bigger separation allows the water to penetrate the $\mu$-cell and wet the surface inside the structure thus increasing the wetting area, while on the other hand a smaller separation between structures give place to no well-define micro-cells.

The pillar-like structures made by means of DLIP were also highly hydrophilic immediately after the laser process, recording a SCA of $\sim 70^{\circ}$ (Fig. 8c). Finally, the hierarchical structures made by the combination of the two laser micro-structuring techniques (DLW + DLIP) were also highly hydrophilic after the laser treatment, but became hydrophobic afterwards, recording a SCA value higher than $110^{\circ}$ (Fig. 8d) improving the C.A. value on comparison with the DLW and DLIP structures separately. This increase on the C.A. must be related with the presence of the hierarchical structures if the Cassie-Baxter model is consider. The combination of both structures (DLW + DLIP) may allow the surface to make a transition from the Wenzel state into a Cassie-Baxter state due to the presence of the hierarchical structures created, thus reducing the area of the liquid-solid interface.

\section{Conclusions}

Multiple-scale periodic surface patterns were fabricated on Ti-6Al-4V alloy combining two laser micro-structuring techniques. Nanosecondpulsed Direct Laser Writing (DLW) has been employed on Titanium alloy (Ti-6Al-4V) in order to create micro-cells ( $50 \mu \mathrm{m}$ width, 4-10 $\mu \mathrm{m}$ depth and $4-10 \mu \mathrm{m}$ high). A high presence of melting, due to the prevalent thermal ablation process, contributed to the formation of the microwalls. The structuring resulted in the creation of squared areas ( $\mu$ cells) regularly distributed on the sample surface. After that, picosecondpulsed Direct Laser Interference Patterning (DLIP) was used in order to create micro-structures with a period of $2.6 \mu \mathrm{m}$. The structuring, mainly free of thermal effects, has been applied in order to create pillars with a height of $1.1 \mu \mathrm{m}$. The small structures created by DLIP have been applied within each bigger $\mu$-cell created by DLW. The resulting structures present a double-scale structure, similar to examples present in nature, and are a promising texturing for super-hydrophobic surfaces. As a consequence of the ultra-short pulses regime and the high overlap between each pulses, the ps-DLIP treatment produced also LIPSS features within 

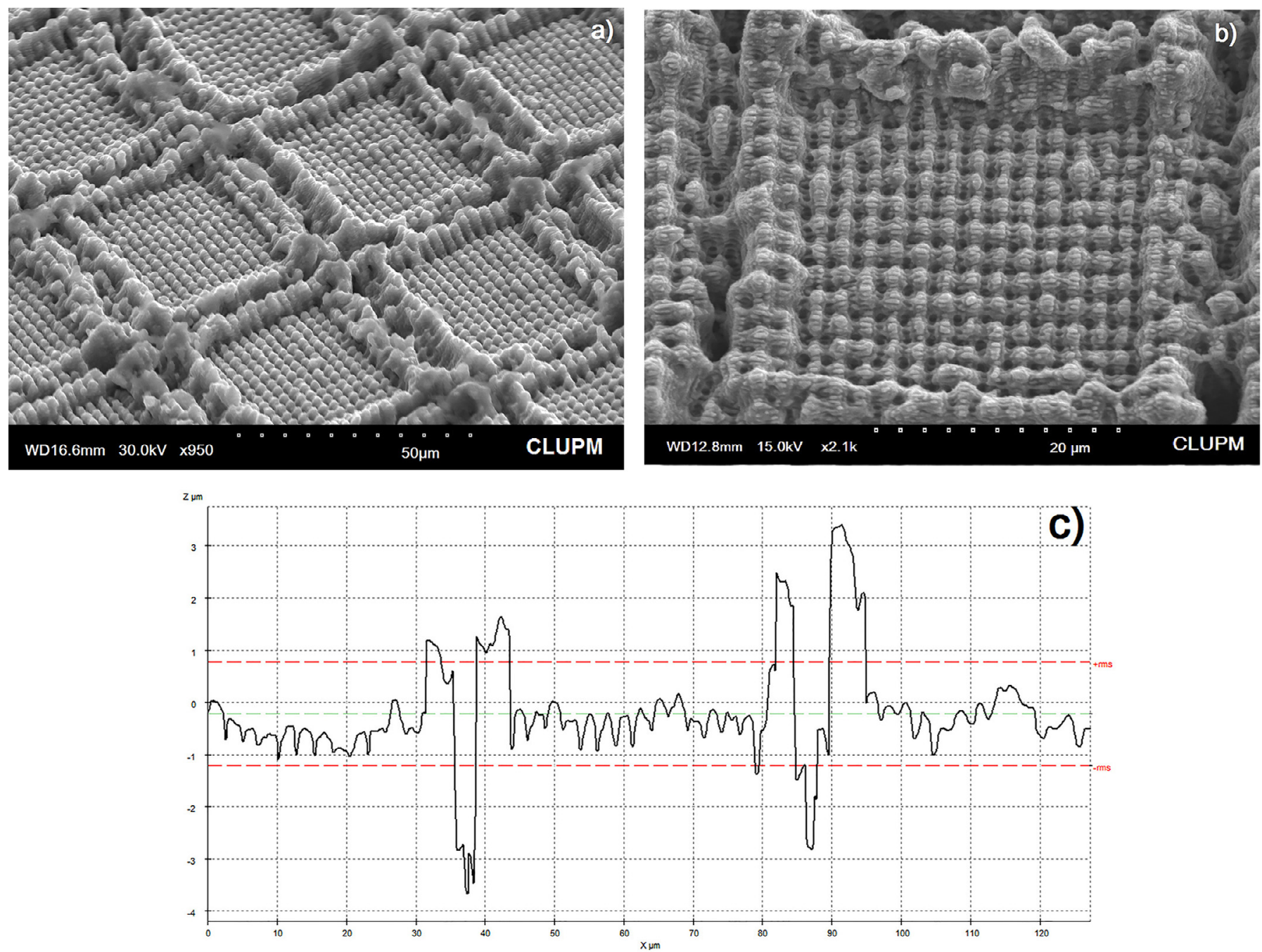

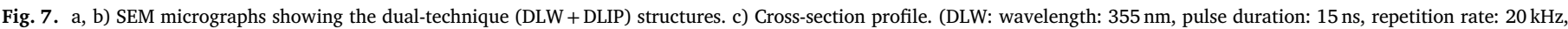

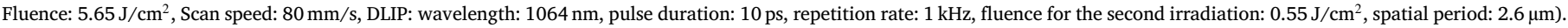

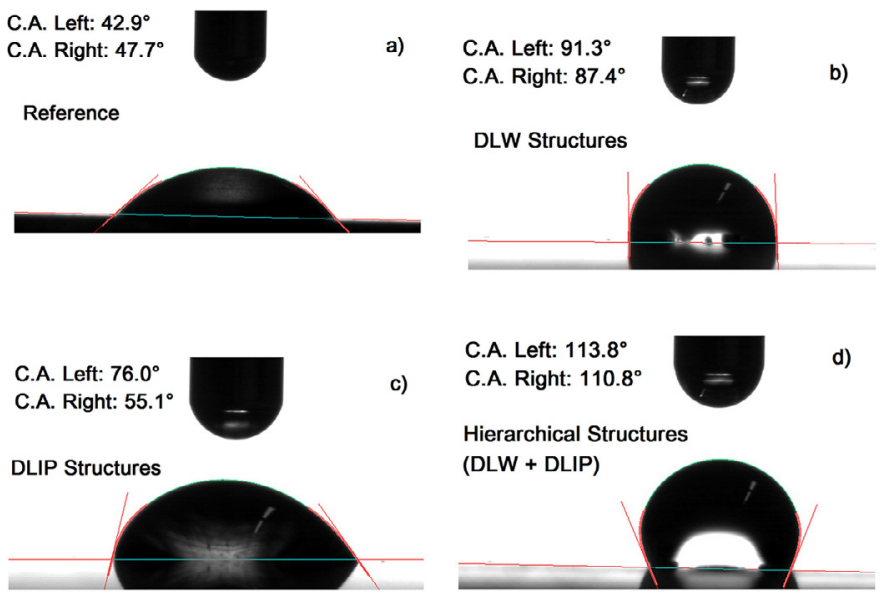

Fig 8. Contac angle measurements: a) Reference, b) DLW $\mu$-cell structures, c) DLIP structures and d) Hierarchical Structures (DLIP + DLW + LIPSS).

each interference maxima during giving place to a triple hierarchical periodic surface structure. The possibility to control the creation of small features like LIPSS, expands the range of applicable techniques available to fabricate hierarchical structures. Additionally, Static Contact An- gle measurements were made in order to obtain a preliminary analysis on the wettability behavior of the structures created on this work. The DLW and the DLIP separately shows a hydrophilic behavior. Hierarchical structures were hydrophobic and recorded a SCA value over $110^{\circ}$, thus allowing the creation of hydrophobic surface that present a potential application on wettability technology.

\section{Acknowledgments}

This work was carried out in the framework of the LASER4FUN project (http://www.laser4fun.eu), which has received funding from the European Union's Horizon 2020 research and innovation programme under the Marie Skłodowska-Curie grant agreement No 675063. The work of A. L. is also supported by the German Research Foundation (DFG) under Excellence Initiative program by the German federal and state governments to promote top-level research at German universities.

\section{References}

[1] Zorba V, Boukos N, Zergioti I, Fotakis C. Ultraviolet femtosecond, picosecond and nanosecond laser microstructuring of silicon: structural and optical properties. Appl Opt 2008;47:1846-50. doi:10.1364/AO.47.001846.

[2] Hwang TY, Vorobyev AY, Guo C. Surface-plasmon-enhanced photoelectron emission from nanostructure-covered periodic grooves on metals. Phys Rev B 2009;79:1-4. doi:10.1103/PhysRevB.79.085425.

[3] Jagdheesh R, Ocaña JL. One-step generation of ultrahydrophobic aluminum surface patterns by nanosecond lasers. Proceeding LIM; 2015. 
[4] Chen F, Zhang D, Yang Q, Yong J, Du G, Si J, et al. Bioinspired wetting surface via laser microfabrication. ACS Appl Mater Interfaces 2013;5:6777-92. doi:10.1021/am401677z.

[5] Barthlott W, Neinhuis C. Purity of the sacred lotus, or escape from contamination in biological surfaces. Planta 1997;202:1-8. doi:10.1007/s004250050096.

[6] Feng L, Zhang Y, Xi J, Zhu Y, Wang N, Xia F, et al. Petal effect: a superhydrophobic state with high adhesive force. Langmuir 2008;24:4114-19. doi:10.1021/la703821h

[7] Ghiradella H, Radigan W. Collembolan cuticle: wax layer and antiwetting properties. J Insect Physiol 1974;20:301-6. doi:10.1016/0022-1910(74)90062-6.

[8] Zheng Y, Gao X, Jiang L. Directional adhesion of superhydrophobic butterfly wings. Soft Matter 2007;3:178. doi:10.1039/b612667g.

[9] Sun W, Neuzil P, Kustandi TS, Oh S, Samper VD. The nature of the gecko lizard adhesive force. Biophys J 2005;89:L14-17. doi:10.1529/biophysj.105.065268.

[10] Liu K, Du J, Wu J, Jiang L. Superhydrophobic gecko feet with high adhesive forces towards water and their bio-inspired materials. Nanoscale 2012;4:768. doi:10.1039/c1nr11369k

[11] Liu K, Yao X, Jiang L. Recent developments in bio-inspired special wettability. Chem Soc Rev 2010;39:3240-55. doi:10.1039/b917112f.

[12] Liu K, Jiang L. Bio-inspired design of multiscale structures for function integration. Nano Today 2011;6:155-75. doi:10.1016/j.nantod.2011.02.002.

[13] Ghio S, Paternoster G, Bartali R, Belluti P, Boscardin M, Pugno NM. Fast and large area fabrication of hierarchical bioinspired superhydrophobic silicon surfaces. J Eur Ceram Soc 2015;36:2363-9. doi:10.1016/j.jeurceramsoc.2016.01.041.

[14] Mohamed AMA, Abdullah AM, Younan NA. Corrosion behavior of superhydrophobic surfaces: a review. Arab J Chem 2015;8:749-65. doi:10.1016/j.arabjc.2014.03.006.

[15] Feng L, Zhang H, Wang Z, Liu Y. Superhydrophobic aluminum alloy surface: fabrication, structure, and corrosion resistance. Colloids Surfaces A Physicochem Eng Asp 2014;441:319-25. doi:10.1016/j.colsurfa.2013.09.014.

[16] Zhang F, Chen S, Dong L, Lei Y, T Liu, Yin Y. Preparation of superhydrophobic films on titanium as effective corrosion barriers. ACS Appl Mater Interfaces 2009;1:11503. doi:10.1021/am900100q.

[17] Martínez-Calderon M, Rodríguez A, Dias-Ponte A, Morant-Miñana MC, GómezAranzadi M, Olaizola SM. Femtosecond laser fabrication of highly hydrophobic stainless steel surface with hierarchical structures fabricated by combining ordered microstructures and LIPSS. Appl Surf Sci 2016;374:81-9. doi:10.1016/j.apsusc.2015.09.261.

[18] Rukosuyev MV, Lee J, Cho SJ, Lim G, Jun MBG. One-step fabrication of superhydrophobic hierarchical structures by femtosecond laser ablation. Appl Surf Sci 2014;313:411-17. doi:10.1016/j.apsusc.2014.05.224

[19] Bizi-Bandoki P, Benayoun S, Valette S, Beaugiraud B, Audouard E. Modifications of roughness and wettability properties of metals induced by femtosecond laser treatment. Appl Surf Sci 2011;257:5213-18. doi:10.1016/j.apsusc.2010.12.089.

[20] Niinomi M. Mechanical properties of biomedical titanium alloys. Mater Sci Eng A 1998;243:231-6. doi:10.1016/S0921-5093(97)00806-X

[21] Niinomi M. Mechanical biocompatibilities of titanium alloys for biomedical applications. J Mech Behav Biomed Mater 2008;1:30-42. doi:10.1016/j.jmbbm.2007.07.001.

[22] Li SY, Li Y, Wang J, Nan YG, Ma BH, Liu ZL, et al. Fabrication of pinecone-like structure superhydrophobic surface on titanium substrate and its self-cleaning property. Chem Eng J 2016;290:82-90. doi:10.1016/j.cej.2016.01.014.

[23] Yang Y, Yang J, Liang C, Wang H, Zhu X, Zhang N. Surface microstructuring of Ti plates by femtosecond lasers in liquid ambiences: a new approach to improving biocompatibility. Opt Express 2009;17:21124-33. doi:10.1364/OE.17.021124.

[24] Radmanesh M, Kiani A. Effects of laser pulse numbers on surface biocompatibility of titanium for implant fabrication. J Biomater Nanobiotechnol 2015;6:168-75. doi:10.4236/jbnb.2015.63017.

[25] Fadeeva E, Truong VK, Stiesch M, Chichkov BN, Crawford RJ, Wang J, et al. Bacterial retention on superhydrophobic titanium surfaces fabricated by femtosecond laser ablation. Langmuir 2011;27:3012-19. doi:10.1021/la104607g.

[26] Dumont T, Lippert T, Wokaun A, Leyvraz P. Laser writing of 2D data matrices in glass. Thin Solid Films 2004;453-454:42-5. doi:10.1016/j.tsf.2003.11.148.

[27] Selimis A, Mironov V, Farsari M. Direct laser writing: Principles and materials for scaffold 3D printing. Microelectron Eng 2014;132:83-9. doi:10.1016/j.mee.2014.10.001.

[28] Zhou Y, Bao O, Varghese B, Tang LAL, Tan CK, Sow CH, et al. Microstructuring of graphene oxide nanosheets using direct laser writing. Adv Mater 2010;22:67-71. doi:10.1002/adma.200901942

[29] Jagdheesh R, García-Ballesteros JJ, Ocaña JL. One-step fabrication of near superhydrophobic aluminum surface by nanosecond laser ablation. Appl Surf Sci 2016;374:2-11. doi:10.1016/j.apsusc.2015.06.104.

[30] Jagdheesh R, Diaz M, Ocaña JL. Bio inspired self-cleaning ultrahydrophobic aluminium surface by laser processing. RSC Adv 2016;6:72933-41. doi:10.1039/c6ra12236a.

[31] Lasagni AF, Beyer E. Fabrication of periodic submicrometer and micrometer arrays using laser interference-based methods. Elsevier Ltd.; 2015. doi:10.1016/B978-1-78242-074-3.00017-9.

[32] Lasagni AF, Roch T, Berger J, Kunze T, Lang V, Beyer E. To use or not to use (direct laser interference patterning), that is the question. SPIE 9351; 2015. doi:10.1117/12.2081976.
[33] Demuth C, Bieda M, Lasagni AF, Mahrle A, Wetzig A, Beyer E. Thermal simulation of pulsed direct laser interference patterning of metallic substrates using the smoothed particle hydrodynamics approach. J Mater Process Technol 2012;212:689-99. doi:10.1016/j.jmatprotec.2011.10.023.

[34] Guenther D, Valle J, Burgui S, Gil C, Solano C, Toledo-Arana A, et al. Direct laser interference patterning for decreased bacterial attachment. In: Proc. SPIE - Int. Soc. Opt. Eng., vol. 9736; 2016. p. 1-9. doi:10.1117/12.2216065.

[35] Bieda M, Beyer E, Lasagni AF. Direct fabrication of hierarchical microstructures on metals by means of direct laser interference patterning. J Eng Mater Technol 2010;132:31015. doi:10.1115/1.4001835.

[36] Bieda M, Siebold M, Lasagni AF. Fabrication of sub-micron surface structures on copper, stainless steel and titanium using picosecond laser interference patterning. Appl Surf Sci 2016;387:175-82. doi:10.1016/j.apsusc.2016.06.100.

[37] Soldera M, Taretto K, Berger J, Lasagni AF. Potential of photocurrent improvement in $\mu \mathrm{c}$-Si:H solar cells with TCO substrates structured by direct laser interference patterning. Adv Eng Mater 2016;18:1674-82. doi:10.1002/adem.201600225.

[38] Lasagni AF, Roch T, Langheinrich D, Bieda M. Large area direct fabrication of periodic arrays using interference patterning. Phys Procedia 2011;12:214-20. doi:10.1016/j.phpro.2011.03.12.

[39] Alamri S, Lasagni AF. Development of a general model for direct laser interference patterning of polymers. Opt Express 2017;25:9603. doi:10.1364/OE.25.009603.

[40] Eckhardt S, Siebold M, Lasagni AF. Laser microstructured metal thin films as promising alternative for indium based transparent electrodes. Opt Express 2016;24:A55368. doi:10.1364/OE.24.00A553.

[41] Roch T, Klein F, Guenther K, Roch A, Mühl T, Lasagni A. Laser interference induced nano-crystallized surface swellings of amorphous carbon for advanced micro tribology. Mater Res Express 2014;1:35042. doi:10.1088/2053-1591/1/3/035042.

[42] Guo R, Yuan D, Das S. Large-area microlens arrays fabricated on flexible polycarbonate sheets via single-step laser interference ablation. J Micromech Microeng 2011;21:15010. doi:10.1088/0960-1317/21/1/015010.

[43] Guo L, Jiang HB, Shao RQ, Zhang YL, Xie SY, Wang JN, et al. Two-beam-laser interference mediated reduction, patterning and nanostructuring of graphene oxide for the production of a flexible humidity sensing device. Carbon 2012;50:1667-73. doi:10.1016/j.carbon.2011.12.011.

[44] Stankevicius E, Balciunas E, Malinauskas M, Raciukaitis G, Baltriukiene D, Bukelskiene V. Holographic lithography for biomedical applications. In: Proc. SPIE - Int. Soc. Opt. Eng., vol. 8433; 2012.

[45] Marczak J, Kusiński J, Major R, Rycyk A, Sarzyński A, Strzelec M, et al Laser interference patterning of diamond-like carbon layers for directed migration and growth of smooth muscle cell depositions. Opt. Appl. 2014;44:575-86. doi:10.5277/oa140408.

[46] Czyż K, Marczak J, Major R, Mzyk A, Rycyk A, Sarzyński A, et al. Selected laser methods for surface structuring of biocompatible diamond-like carbon layers. Diam Relat Mater 2016;67:26-40. doi:10.1016/j.diamond.2016.01.013.

[47] Lang V, Roch T, Lasagni AF. High-speed surface structuring of polycarbonate using direct laser interference patterning: toward $1 \mathrm{~m}^{2} \mathrm{~min}^{-1}$ fabrication speed barrier. Adv Eng Mater 2016;18:1342-8. doi:10.1002/adem.201600173.

[48] Lang V, Roch T, Lasagni AF. World record in high speed laser surface microstructuring of polymer and steel using direct laser interference patterning. In: Proc. SPIE Int. Soc. Opt. Eng., vol. 9736; 2016. p. 1-8. doi:10.1117/12.2216203.

[49] Skolski JZP, Römer GRBE, Obona JV, Ocelik V, Huis In 't Veld AJ, De Hosson JTM. Laser-induced periodic surface structures: fingerprints of light localization. Phys Rev B 2012;85:1-9. doi:10.1103/PhysRevB.85.075320.

[50] Bonse J, Krüger J, Höhm S, Rosenfeld A. Femtosecond laser-induced periodic surface structures. J Laser Appl 2012;24:42006. doi:10.2351/1.4712658.

[51] Camacho-López S, Evans R, Escobar-Alarcón L, Camacho-López MA, CamachoLópez MA. Polarization-dependent single-beam laser-induced grating-like effects on titanium films. Appl Surf Sci 2008;255:3028-32. doi:10.1016/j.apsusc.2008.08.085.

[52] Tan B, Venkatakrishnan K. A femtosecond laser-induced periodical surface structure on crystalline silicon. J Micromech Microeng 2006;16:1080-5. doi:10.1088/0960-1317/16/5/029

[53] Young JF, Sipe JE, Preston JS, Van Driel HM. Laser-induced periodic surface damage and radiation remnants. Appl Phys Lett 1982;41:261-4. doi:10.1063/1.93494.

[54] Bonse J, Rosenfeld A, Krüger J. On the role of surface plasmon polaritons in the formation of laser-induced periodic surface structures upon irradiation of silicon by femtosecond-laser pulses. J Appl Phys 2009;106. doi:10.1063/1.3261734.

[55] Römer G.R.B.E., Skolski J.Z.P., Oboňa J.V., Ocelík V., de Hosson J.T.M., Huis in 't Veld A.J. Laser-induced periodic surface structures, modeling, experiments, and applications 2014;8968:89680D. doi:10.1117/12.2037541.

[56] Skolski JZP, Römer GRBE, Huis in't Veld AJ, Mitko VS, Obona JV, Ocelik V, et al. Modeling of laser induced periodic surface structures. J Laser Micro Nanoeng 2010;5. doi:10.2961/jlmn.2010.03.0015.

[57] Skolski JZP, Römer GRBE, Vincenc Obona J, Huis In 'T Veld AJ. Modeling laserinduced periodic surface structures: finite-difference time-domain feedback simulations. J Appl Phys 2014;115. doi:10.1063/1.4867759.

[58] Aguilar-Morales AI, Alamri S, Lasagni AF. Micro-fabrication of high aspect ratio periodic structures on stainless steel by picosecond direct laser interference patterning. J Mater Process Technol 2017 (Submitted). 


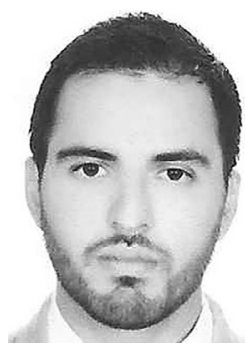

Daniel Huerta Murillo received his Bachelor's Degree (2013) in Physics from the Autonomous University of Zacatecas (UAZ). He earned his M.Sc. (2015) in Optics at the Ensenada Center for Scientific Research and Higher Education (CICESE). His thesis gave him experience laser materials processing by doing an experimental study of the formation of nano-structures on metals after laser irradiation, which were characterized by SEM, AFM and Raman Spectroscopy. Currently (2016) he is a Ph.D. student and an early stage researcher (ESR) at the Laser Centre of the Polytechnical University of Madrid (CL-UPM) as a member of the LASER4FUN Marie Sklodowska Curie project.
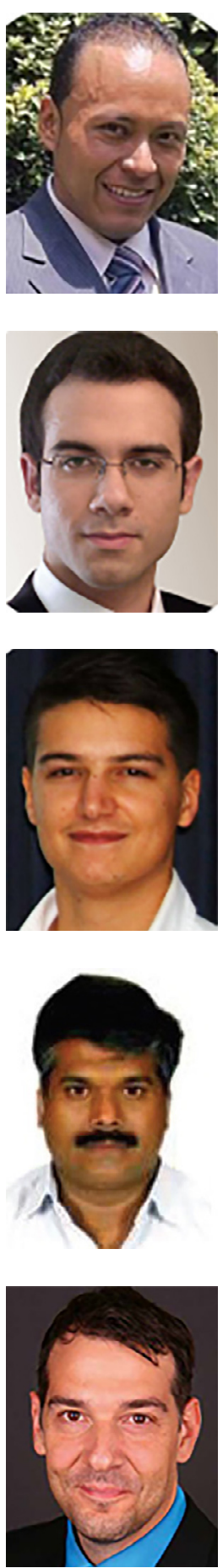

R. Jagdheesh, was awarded his M.Sc. (1998) and Ph.D. (2005) in Physics from Bharathidasan University, India. After the Ph.D., he worked as postdoctoral fellow at Raja Ramanna Centre for Advanced Technologies in India, ENSMA CNRS in France, University of Twente in Netherlands, and University of New South Wales, Australia before he joined the CLUPM, Polytechnical University of Madrid in Spain. His research is focused on laser cladding, laser surface alloying and laser micromachining for surface functionalization. He has published more than 35 research papers in journals and conference proceedings.

o. I Aguilar-Morales obtained a B.Eng. degree in industrial robotics at ESIME-IPN in 2009, M.Sc. in advanced technology (photonics) from CIITEC-IPN in 2015 (both in Mexico city), the research was carried out in the laser-obsidian interaction in photomechanical ablation. He is currently a Ph.D. student in the laser 4 fun project, his research activities are focused on surface material structuring by the Direct Laser Interference Patterning at Fraunhofer IWS institute Dresden, Germany. His main research interests include superhydrophobic properties, antiicing and friction reduction.

Sabri Alamri is a doctoral researcher in the Group of Surface Functionalization at the Fraunhofer IWS (Germany), and at the Institute for Manufacturing Technology of the Technical University of Dresden since 03/2016. Sabri completed his studies in Materials Science and Technologies at University of Bari (Italy) in 07/2015. During the bachelor's degree he worked on Self-mixing Interferometry, while for the master thesis he worked on nanoscale analyses with AFM and Raman Spectroscopy. Currently his research interests lie in the area of Laser surface structuring, in particular in Direct Laser Interference Patterning of polymers and modeling of laser-matter interaction processes.

J.T. Cardoso is a Portuguese Ph.D. researcher on the LASER4FUN Marie Sklodowska Curie project in the Laser Centre of the Universidad Politécnica de Madrid since March 2016. Natural from Barcelos, in Portugal, he was born in 1991 and finished his integrated Master's degree in Physics Engineering at the Faculty of Science of the University of Porto (FCUP) on October 2015. In his master thesis he designed and developed Nanoscaled Piezoelectric Energy Harvesters, guided by the principle of converting external mechanical movements into electricity by the deformation of the device.

Andrés F. Lasagni received in 2002 his M.S. degree in Chemical Engineering from the Comahue National University (Argentina). From 2003 to 2005 he carried out his Ph.D. at Saarland University (Germany) and in 2007-2008 he conducted a postdoctoral stay at the Georgia Institute of Technology and the University of Michigan. Since 2008 he is group leader at the Fraunhofer IWS and since 2012 is professor at the Technische Unversität Dresden (Germany). A.L is author/coauthor of more than 150 publications and has been awarded with several prizes including the German High Tech Champion in Photovoltaic and the Green Photonic Award. 


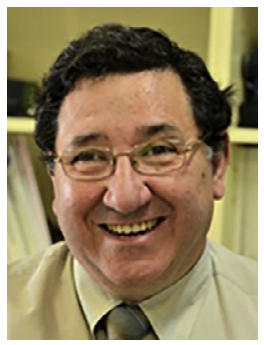

Jose L. Ocaña was awarded his M.Sc. (1979) and a Ph.D. (1982) in Industrial Engineering at the Polytechnical University of Madrid (Spain). He is Chair Professor of Mechanical Engineering at the ETSII-UPM School of Engineering. He is responsible for coordination of worldwide R\&D initiatives in the field of scientific and industrial applications of high-power lasers. Author/coauthor of more than 150 scientific papers and more than 200 communications in the field of laser technology and applications. Former Chairman of EULASNET II (Eureka Umbrella Network for Laser Tex|chnology and Applications). Member of the Executive Board of the European Laser Institute (ELI). 